\title{
Time-Dependent Event-Tree Method for Fire Risk Analysis: Tentative Results
}

\author{
TIMO KORHONEN, JUKKA HIETANIEMI, DJEBAR BAROUDI, and \\ MATTI KOKKALA \\ V T T Technical Research Centre of Finland \\ P.O. Box 1803, FIN-02044 VTT, Finland
}

\begin{abstract}
A fire risk analysis method based on the use of time-dependent event trees is presented. To concretize the method, a simple example case, a property-loss risk analysis for a fire in a one-storey industrial hall, is presented. The method incorporates explicitly the time dependence of the fire and its consequences. This is achieved so that the fire incident is divided to time intervals and the events within each time interval are analyzed with a separate event tree. The event trees form the basic structure of the analysis and it is shown here how to connect them to a description of the evolution of the fire. This description can be expressed as a stochastic Markov process and it is shown how to link the branching probabilities of the event trees to the transfer matrices of the Markov chain.
\end{abstract}

KEYWORDS: Fire Safety, Risk Assessment, Time Dependent, Event Tree, Stochastic Process, Markov Chain, Monte Carlo

\section{INTRODUCTION}

The development of fire safety engineering into a well-defined engineering discipline has enabled administrations around the world to make a change towards performance-based fire safety regulations. In performance-based design, the most rigorous way to assess the acceptability of a design solution is to use quantitative risk analysis, which, in addition to quantifying the resulting risk level, can also identify the critical factors of the design.

In addition to the use in design of buildings and constructions, fire risk analysis can also be used to examine the fire regulations to establish whether the requirements are in accordance with their role in reducing of the fire risks. The method presented in this article is a tool for such a study with focus on the fire resistance requirements.

The method lays emphasis to the fire resistance requirements of load-bearing structures. The balance between the level of fire resistance requirements and their impact on the fire risks are of great economic concern because they often are a significant factor influencing the selection of construction materials 


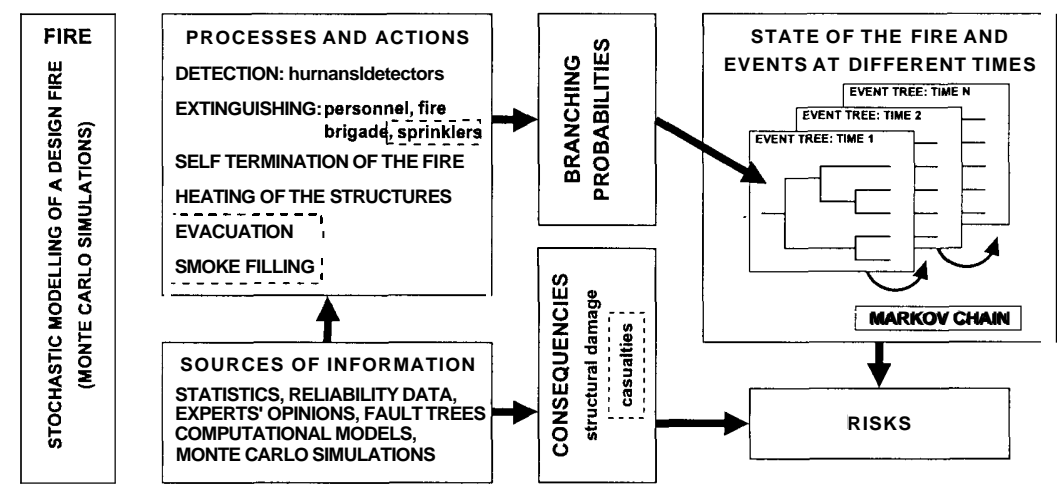

Figure 1: Overview of the method. The items encircled by dashed line are still being developed and thus are not included in the presentation given in this article.

and construction costs. Presently in Europe, the fire resistance requirements vary considerably between different countries, or even between different authorities within one country, often for no obvious reason. The regulatory diversity derives from the fact that the requirements are based more on, e.g., historical development rather than science and also the rationale behind the national requirements may vary considerably, which implies that there is a definite need to establish a rational risk-motivated basis for the fire resistance requirements.

There exist several fire risk analysis methods, such as CRISP [I],FiRECAM ${ }^{\mathrm{TM}}$ [2], and CESARE-RISK [3] to mention some of the recent methods. However, mainly due to the strong fire-resistance orientation of the end-use of the particular application related to the present work, it was decided not to resort to use of any of the above mentioned methods, but to develop a new method. The method is not yet completed, but is in a state of continuous development. For example, a sufficiently versatile and reliable evacuation model is not yet implemented. Thus, only the potential consequences to property arising from failure of structures are considered here. The presentation is made concrete by applying the model to a target building, a single-storey industrial building.

\section{OVERVIEW OF THE METHOD AND ITS APPLICATION}

The time-dependent event tree model and the other methods used to examine the risks of a chosen fire scenario are shown schematically in Fig. 1. The basic idea of the model is to analyze the fire incident by using the stochastic design fire concept developed in this work. Some of the events and processes investigated during a fire are described with computational models. The inherent variations in some processes are modeled by Monte Carlo simulations. The fire incident is divided to a relatively small number of discrete time intervals. At each time interval an event tree is constructed to describe the evolution of the fire and the potential actions (by humans or automatic systems) to 


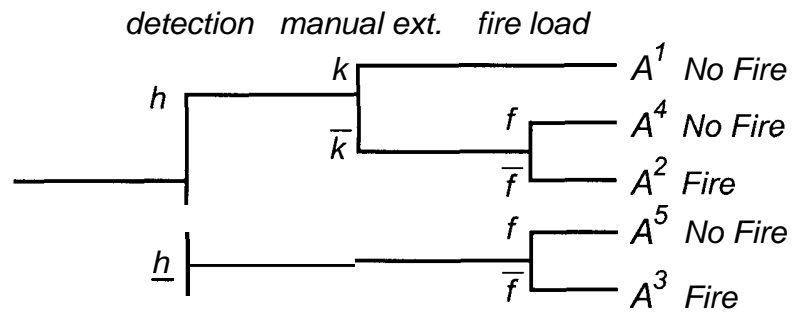

Figure 2: The basic event tree used to analyze the fire in the target building at different times. Shown are also the labels of the time dependent probabilities associated with the branching points. A bar means the complement of the probability, e.g., $h=1-h$.

detect and fight the fire. The event trees are accompanied with analysis of the consequences of the fire at each time interval.

The example event tree structure used in this work to concretize the method is shown in Fig. 2. The branches of the event trees correspond to the following three events: detection of the fire (probability $h$ ), manual extinguishing (probability $k$ ), and burning out of the fire load (probability $f$ ). The system can be only in five mutually exclusive states at each time interval $t$ :

$A_{t}^{1}$ : No fire (detected, manual extinguishment)

$A_{t}^{2}$ : $\quad$ Fire (detected)

$A_{t}^{3}$ : $\quad$ Fire (undetected)

$A_{t}^{4}$ : No fire (detected, fire load has burnt out)

$A_{t}^{5}$ : No fire (undetected, fire load has burnt out)

The system is at state $A^{3}$ when the fire breaks out at time $t_{0}=0$. Note, that in the states $A^{2}$ and $A^{4}$ there has been at least one failed extinguishment attempt, because extinguishment actions are supposed to be started as soon as the fire is detected.

The event trees that are describing the progress of a fire at a given time interval are subjected to a condition that the fire is not extinguished before the interval. The determination of the time evolution of the fire can be carried out either by employing conditional probabilities or by a state transition process description of the system, which can be described as a Markov process. This process is shown schematically in Fig. 3. The transition probabilities corresponding to a transition between successive time intervals are also marked in the figure.

For example, one can end up in the state $A^{4}$ (fire is detected, manual extinguishment has failed, fire load has ended) by two different ways: 1)from state $A^{3}$ by detecting the fire, failing manual extinguishment, and by burning out the fire load giving a transition probability $h \bar{k} \bar{f} ; 2$ ) from state $A^{2}$ by burning out the fire load which has a transition probability $\bar{f}$. Similarly all the other transition probabilities between the different states shown in Fig. 3 can be 


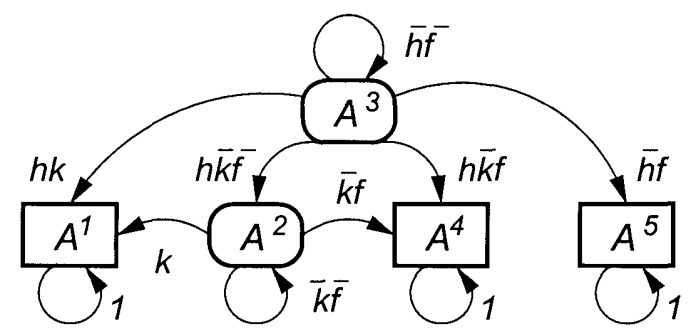

Figure 3: The state-transition process described by the time-dependent event tree. When the fire starts (time zero) the system is in the state $A^{3}$. States denoted by the sharp rectangles are final states of the system, i.e. states where the fire is extinguished. The fire is still burning in states which are denoted by the rounded rectangles. The time-dependent transition probabilities are related to the branching probabilities of the event trees as shown.

found. Note, that there is only one extinguishment trial allowed during each time interval and that this attempt is "instantaneous",i.e. if the attempt is successful then there fire is already regarded extinguished at the beginning of the next time interval.

\section{Branching probabilities of the event trees}

The branching probabilities of the event trees are calculated by using design fires. A design fire is characterized by the time evolution of its heat release rate (HRR) .In this work, a design fire is considered to be of stochastic nature, where its growth, maximum size, and duration are varying within some specific ranges (see Fig. 4a). These input design fires to the system are assumed to be freely developing fires, i.e., there are no extinguishing attempts performed (neither by humans nor by automatic devices) [4].

The probability of detection by senses depends on the fire size at a given time. For a $t^{2}$-fire growing with normal speed (growth time $300 \mathrm{~s}$ ) detection probability is assumed be $0,0.5$, and 0.9 after 5,10 , and 15 minutes from the ignition, respectively (the times correspond to fire sizes $1 \mathrm{MW}, 4 \mathrm{MW}$, and $9 \mathrm{MW})$. All fires are assumed to be detected during the first 30 minutes of the fire. These values are obtained by heuristic reasoning rather than estimation based on exact data. The values are then interpolated and the probability of fire detection by senses as a function of time is calculated by a Monte Carlo average over the stochastic HRR curves.

The probability of automatic detection by heat detectors is modeled by calculating the activation times of the detectors. The gas temperature and flow velocity in the ceiling jet are modeled using Alpert's empirical correlations [5] and the activation time of the detector is obtained by solving the differential equation describing its heating. This is done for each simulated HRR curve and thus, a probability distribution of the automatic detection is obtained. 

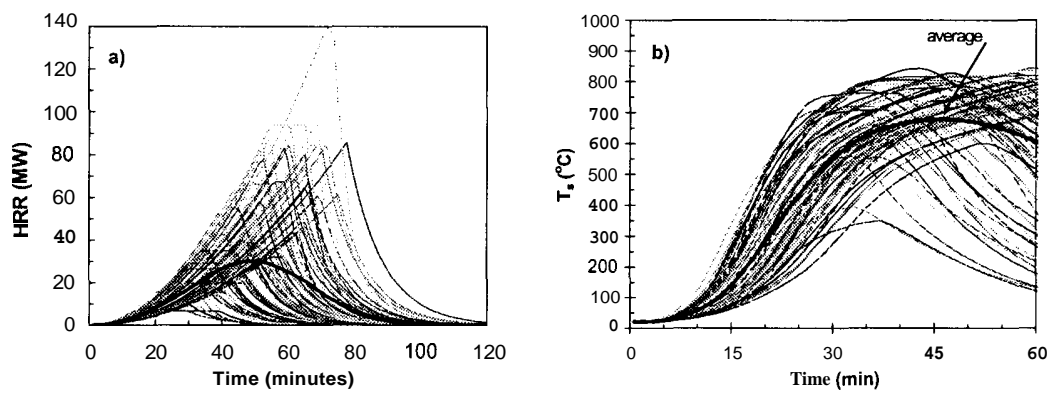

Figure 4: a) Stochastic design fires used in this work, b) Corresponding steel temperatures calculated for an I-beam (section factor $F / V=150 \mathrm{~m}^{-1}$ ).

The probabilities of the burning out of the fire load are obtained from Monte Carlo simulations by counting the fraction of fires whose HRR has started to decrease from its peak value. In this work, the fire load is supposed to be finished when the HRR of a freely developing design fire has decayed to half of its peak value.

The probabilities of the manual extinguishing (both by personnel and the fire brigade) are calculated similarly to the probability of detection by senses, i.e., values for a few fire sizes are obtained via reasoning based on expert judgments and then a Monte Carlo average is formed. If the extinguishing is done by the personnel, the values 0.9 and 0.5 are used for fire sizes $1 \mathrm{MW}$ and $4 \mathrm{MW}$, respectively. For the fire brigade the values $0.99,0.8$, and 0.1 are used for fire sizes $9 \mathrm{MW}, 15 \mathrm{MW}$, and $36 \mathrm{MW}$, respectively.

\section{Structural failure}

Heating of structures is modeled using the localized fire exposure description by Hasemi et al. [6] revised by an additional parameter introduced by Myllymaki and Kokkala [7] to describe the proportions of convective and radiative heat transfer. An example of the calculated steel temperatures is shown in Fig. 4b for a bended simply supported I-beam (section factor $\mathrm{F} / \mathrm{V}=1501 / \mathrm{m}$ ). In this paper, a simple structural failure mode is used. The steel beams are assumed to fail if their temperature rises above a critical temperature of $600^{\circ} \mathrm{C}$. The probabilities of structural damage (denoted $v$ in the following) as a function of time is then simply obtained by tabulating the times when the steel temperature reaches the critical value in each of the calculated curves. 


\section{APPLICATION OF THE METHOD TO THE EXAMPLE CASE}

\section{Target building and fire scenario}

The building examined is a one-storey industrial building with floor area of $5040 \mathrm{~m}^{2}$ and height of $8 \mathrm{~m}$. The load-bearing structures (columns and beams) are made of unprotected steel and the walls of lightweight concrete. The hall is practically a single open space. As an example, we considerer only fires igniting during the afternoons-evenings of working days $\left(2^{\mathrm{pm}}-10^{\mathrm{pm}}\right)$, when there are only about 5 occupants in the building.

In this study, the main emphasis has been on the ability of the load-bearing structures to carry their load. The most severe fire scenario for this purpose is a large powerful fire which is heating the structures strongly. The selection of design fires used in this work is based on the study of Paavilainen [8], where she concludes that the burning of empty wooden pallets stored in piles represents a severe fire scenario in the target building. Due to the low fire load and the large size of the building as compared to conceivable sizes of fires, the probability of a flashover is negligible, and thus localized fires are chosen as the example fire scenario studied here. Fires which evolve to a flashover could as easily be studied using the same event trees but then the probabilities of the branches should be calculated differently as compared to localized fires.

According to Babrauskas [9], the maximum heat release rate (HRR) of burning wood pallets can be evaluated from the properties of the pallet pile, i.e., its floor area, height, wood moisture, and effective heat of combustion. The variability of the design fire is modeled by treating these parameters as well as the growth time and decay time constant of the fire as a stochastic quantities. In the calculations moisture was fixed to $10 \%$ while all the other parameters were selected from suitable distributions. The time evolution of the fire, which develops freely without external interruptions, is modeled using a $t^{2}$-design fire with the growth time varying randomly between $300 \mathrm{~s}$ and $600 \mathrm{~s}$ and the height of the pallet pile and its floor area varied uniformly between $1-5 \mathrm{~m}$ and $2-20 \mathrm{~m}^{2}$, respectively. As a result we obtain a multitude of HRR curves (see Fig. 4a), from which, e.g., the probability that the fire stops to fire load burn out can be derived.

Only the first 120 minutes of a fire are considered in the risk analysis of the target building. This time period is divided to 6 time intervals, where the end points of the intervals are $t_{1}=5, t_{2}=10, t_{3}=15, t_{4}=30, t_{5}=60$, and $t_{6}=120$ minutes. The event tree structure is shown in Fig. 2 and the branching probabilities of the event trees are summarized in Table 1. Note, that the fire brigade is assumed to appear at the fire location during the third time interval (10-15 $\mathrm{min})$. The driving distance is $7.7 \mathrm{~km}$ and according to Tillander and Keski-Rahkonen [10] the travel time is then just over eight minutes in the Uusimaa region in Southern Finland.

The probability of detection $\left(H_{t}\right)$ and the probability that the fire load has finished $\left(F_{t}\right)$ are tabulated as cumulative probability distributions. (Cumulative distributions are labeled with uppercase letters whereas the branching 
Table 1: The time dependent probabilities associated with the branching points of the event trees. Also shown is the probability of damage to the structures. The symbols used are explained in the text.

\begin{tabular}{lcccccc}
\hline \hline & $5 \mathrm{rnin}$ & $10 \mathrm{rnin}$ & $15 \mathrm{rnin}$ & $30 \mathrm{~min}$ & $60 \mathrm{~min}$ & $120 \mathrm{~min}$ \\
\hline Detection, $H_{t}$ & 0.08 & 0.79 & 0.95 & 1.00 & 1.00 & 1.00 \\
Manual ext., $k_{t}$ & 0.95 & 0.75 & 0.97 & 0.68 & 0.39 & 0.39 \\
No fire load, $F_{t}$ & 0.00 & 0.00 & 0.00 & 0.00 & 0.65 & 1.00 \\
\hline Damaged, $v_{t}$ & 0.00 & 0.00 & 0.00 & 0.35 & 0.93 & 0.93 \\
\hline \hline
\end{tabular}

probabilities with lowercase letters.) Cumulative distributions like these can not be directly used as branching probabilities, because one should use conditional probabilities at the branching points of the time dependent event trees. For example, in order to detect the fire at time interval 5-10 min, it should not be detected during the first 5 minutes. To calculate the conditional probability that the fire is detected, $a_{2}$, during the time interval $t_{1}-t_{2}$ Bayes' theorem should be used:

$$
\begin{aligned}
a_{2} & =P\left[\text { detected } t_{1}-t_{2} \mid \text { not detected } t_{0}-t_{1}\right]=P\left[t_{1,2} \mid \overline{t_{0,1}}\right] \\
& =\frac{P\left[\overline{t_{0,1}} \mid t_{1,2}\right] P\left[t_{1,2}\right]}{P\left[\overline{t_{0,1}} \mid t_{0,1}\right] P\left[t_{0,1}\right]+P\left[\overline{t_{0,1}} \mid t_{1,2}\right] P\left[t_{1,2}\right]+P\left[\overline{t_{0,1}} \mid t_{2, \infty}\right] P\left[t_{2, \infty}\right]} \\
& =\frac{1 \cdot P\left[t_{1,2}\right]}{0 \cdot P\left[t_{0,1}\right]+1 \cdot P\left[t_{1,2}\right]+1 \cdot P\left[t_{2, \infty}\right]}=\frac{H_{2}-H_{1}}{1-H_{1}}=0.77
\end{aligned}
$$

\section{Calculation of the time evolution of the probabilities}

The probabilities of the states $A^{j}$ after the break out of a fire are calculated by using the time dependent branching probabilities of the event trees $h_{t}$, $f_{t}$, and $k_{t}$. The following notation is used for the probabilities of the states: $P\left[A_{t}^{j}\right]$ is the probability that the system is in the state $A^{j}$ at time $t$. At time $t_{0}=0$ the fire ignites and the system is in the state $A^{3}$ (fire, not detected). The probability $P\left[A_{0}^{3}\right]$ is thus equal to one and the probabilities of all the other states are zeros.

At the end point of the first time interval, $t_{1}=5$ minutes, these probabilities can be deduced from the event tree shown in Fig. 2

$$
\begin{aligned}
& P\left[A_{1}^{1}\right]=h_{1} \cdot k_{1} \cdot P\left[A_{0}^{3}\right]=0.08 \cdot 0.95 \cdot 1=0.076 \\
& P\left[A_{1}^{2}\right]=h_{1} \cdot \bar{k}_{1} \cdot \bar{f}_{1} \cdot P\left[A_{0}^{3}\right]=0.08 .0 .05 \cdot 1.00 .1=0.004 \\
& P\left[A_{1}^{3}\right]=\bar{h}_{1} \cdot \bar{f}_{1} \cdot P\left[A_{0}^{3}\right]=0.92 \cdot 1.00 \cdot 1=0.920 \\
& P\left[A_{1}^{4}\right]=h_{1} \cdot \bar{k}_{1} \cdot f_{1} \cdot P\left[A_{0}^{3}\right]=0.08 \cdot 0.05 \cdot 0.00 \cdot 1=0.000 \\
& P\left[A_{1}^{5}\right]=\bar{h}_{1} \cdot f_{1} \cdot P\left[A_{0}^{3}\right]=0.92 \cdot 0.00 .1=0.000
\end{aligned}
$$

The probabilities $P\left[A_{1}^{4}\right]$ and $P\left[A_{1}^{5}\right]$ are zero because the fire load does not burn out during the first 30 minutes. Note, that the sum of the probabilities, $\sum_{j=1}^{5} P\left[A_{1}^{\jmath}\right]$ is equal to one as it should be. 
Table 2: Probabilities of the states at different times. The numbers are shown to a greater accuracy than there are physically significant numbers. In this way, it is demonstrated that the probabilities at each time interval add up to one exactly as they should. The probability $P\left[A_{i}^{5}\right]$ is always zero because the fire is detected by senses before the fire load might end.

\begin{tabular}{ccccccc}
\hline \hline & $5 \mathrm{~min}$ & $10 \mathrm{~min}$ & $15 \mathrm{~min}$ & $30 \mathrm{~min}$ & $60 \mathrm{~min}$ & $120 \mathrm{~min}$ \\
\hline$P\left[A_{i}^{1}\right]$ & 0.076 & 0.6115 & 0.939845 & 0.980750 & 0.988258 & 0.989861 \\
$P\left[A_{i}^{2}\right]$ & 0.004 & 0.1785 & 0.010155 & 0.019250 & 0.004110 & 0 \\
$P\left[A_{i}^{3}\right]$ & 0.920 & 0.2100 & 0.050000 & 0 & 0 & 0 \\
$P\left[A_{i}^{4}\right]$ & 0 & 0 & 0 & 0 & 0.007632 & 0.010139 \\
$P\left[A_{i}^{5}\right]$ & 0 & 0 & 0 & 0 & 0 & 0 \\
\hline
\end{tabular}

At the beginning of the later time intervals the fire could be in some other state than in the state $A^{3}$, so the probabilities of the all five states are calculated for an arbitrary time point $t_{i}$ as (see Fig. 3)

$$
\begin{aligned}
& P\left[A_{i}^{1}\right]=P\left[A_{i-1}^{1}\right]+k_{i} \cdot P\left[A_{i-1}^{2}\right]+h_{i} \cdot k_{i} \cdot P\left[A_{i-1}^{3}\right] \\
& P\left[A_{i}^{2}\right]=\bar{k}_{i} \cdot \bar{f}_{i} \cdot P\left[A_{i-1}^{2}\right]+h_{i} \cdot \bar{k}_{i} \cdot \bar{f}_{i} \cdot P\left[A_{i-1}^{3}\right] \\
& P\left[A_{i}^{3}\right]=\bar{h}_{i} \cdot \bar{f}_{i} \cdot P\left[A_{i-1}^{3}\right] \\
& P\left[A_{i}^{4}\right]=P\left[A_{i-1}^{4}\right]+\bar{k}_{i} \cdot f_{i} \cdot P\left[A_{i-1}^{2}\right]+h_{i} \cdot \bar{k}_{i} \cdot f_{i} \cdot P\left[A_{i-1}^{3}\right] \\
& P\left[A_{i}^{5}\right]=P\left[A_{i-1}^{5}\right]+\bar{h}_{i} \cdot f_{i} \cdot P\left[A_{i-1}^{3}\right],
\end{aligned}
$$

where $t_{i-1}$ is the previous time point (the beginning of the corresponding time interval). These probabilities at the times $t_{i}, \mathrm{i}=1, \ldots, 6$, are tabulated in Table 2.

The probability that there is no fire at time $t$ is the sum of the probabilities of the states $A_{t}^{1}, A_{t}^{4}$, and $A_{t}^{5}$ at this time. The probability that the fire continues according to the input design fire curve at time $t$ is the sum of the probabilities of the states $A_{t}^{2}, A_{t}^{3}, A_{t}^{4}$, and $A_{t}^{5}$. These probabilities are listed in Table 3 together with the probability that the fire will be manually extinguished during a time interval.

\section{Probabilities of consequences}

It is seen from Table 1 that during the first three time intervals (up to 15 minutes) no structural damage is formed, i.e. the steel structures have not yet reached the critical temperature. If there still is fire at 30 minutes after the ignition, then there is a non-zero probability $\left(v_{3}=0.35\right)$ that the structure will have a damage.

The probability $v_{i}$ is a conditional probability. It is the probability of a failure provided that the fire is still design fire at the time $t_{i}$, i.e. extinguishment has not been successful,

$$
v_{i}=P\left[\text { damaged } t_{i} \mid \text { design fire } t_{i}\right]=\frac{P\left[\text { damaged AND design fire } t_{i}\right]}{P\left[\text { design fire } t_{i}\right]} .
$$


Table 3: The state of the fire and the building at different times given as probabilities of the states at the end points of the time intervals. 'Manual Ext.' is the probability that the fire is extinguished manually during the time interval corresponding to the given end point.

\begin{tabular}{lcccccc}
\hline \hline & $5 \mathrm{~min}$ & $10 \mathrm{~min}$ & $15 \mathrm{~min}$ & $30 \mathrm{~min}$ & $60 \mathrm{~min}$ & $120 \mathrm{~min}$ \\
\hline Damaged & 0.000 & 0.000 & 0.000 & 0.007 & 0.014 & 0.014 \\
No fire & 0.076 & 0.612 & 0.940 & 0.981 & 0.996 & 1.000 \\
Design fire & 0.924 & 0.388 & 0.060 & 0.019 & 0.012 & 0.010 \\
\hline Manual ext. & 0.076 & 0.536 & 0.328 & 0.041 & 0.008 & 0.002 \\
\hline \hline
\end{tabular}

The probability that the structure will have a damage at a given time is thus $v_{i} P$ [design fire $t_{i}$ ] if there would be no manual extinguishment actions.

The probability that there will be a structural damage at time $t_{i}$ and that the fire is extinguished manually during the next time interval $t_{i+1}$ is

$$
\begin{aligned}
& \left.P \text { [damaged } t_{i} \text { AND ext. during } t_{i+1}\right] \\
& \quad=P\left[\text { damaged } t_{i} \mid \text { ext. during } t_{i+1}\right] P\left[\text { ext. during } t_{i+1}\right] \\
& \quad=P\left[\text { damaged } t_{i} \mid \text { design fire } t_{i}\right] P\left[\text { ext. during } t_{i+1}\right] \\
& \quad=v_{i} P\left[\text { ext. during } t_{i+1}\right]
\end{aligned}
$$

because if the fire will be manually extinguished during the time interval $t_{i+1}$, it will be a design fire at time $t_{i}$. By summing the above values up to a time $t_{i}$ the probability that the structure is damaged at that time point is obtained. These values are tabulated on the first row of Table 3. The value corresponding to the last time point $120 \mathrm{~min}$, which is about 0.014 , is the overall risk that the building will have a structural damage after the fire incident. By examining the spatial ranges of heat exposures it can be concluded that most of the damages are likely being restricted only to the vicinity of the fire.

\section{FORMULATION OF THE METHOD BY USING MARKOV CHAINS}

By examining the equations above it is noticed that the probabilities of the states at a given time step $t_{i}$ depend only on the probabilities of the previous time step $t_{i-1}$ and on the known time-dependent branching probabilities. If the probabilities of the states are considered to be random variables then the system is a Markov process. A Markov process does not have memory, i.e., its future states are fully described by the present values of the random variables. The process is shown schematically in Fig. 3. The transition probabilities corresponding to a transition $t: t_{i} \rightarrow t_{i+1}$ between different states are also marked in the figure.

Define a state vectors $\mathbf{A}_{i}=\left(A_{i}^{1}, A_{i}^{2}, \ldots, A_{i}^{p}\right)^{T}$ and a probability vectors $\mathbf{P}\left[\mathbf{A}_{i}\right]=\left(P\left[A_{i}^{1}\right], P\left[A_{i}^{2}\right], \ldots, P\left[A_{i}^{p}\right]\right)^{T}, i=1, \ldots, n$. There are $\boldsymbol{p}$ states in the 
system and $n$ time intervals. Using this notation the above process can be written as a Markov chain

$$
\mathbf{P}\left[\mathbf{A}_{i}\right]=\mathcal{M}_{i} \mathbf{P}\left[\mathbf{A}_{i-1}\right], \quad i=1, \ldots, n,
$$

where $M$ is the transition matrix describing the evolution of the system from time $t_{i-1}$ to time $t i$.

In our example case is the system is at the state $A^{3}$ initially, so that the probability vector has a value $\mathbf{P}\left[\mathbf{A}_{0}\right]=(0,0,1,0,0)^{T}$ at time zero. Equation (14) reads as

$$
\left(\begin{array}{c}
P\left[A_{i}^{1}\right] \\
P\left[A_{i}^{2}\right] \\
P\left[A_{i}^{3}\right] \\
P\left[A_{i}^{4}\right] \\
P\left[A_{i}^{5}\right]
\end{array}\right)=\left(\begin{array}{lllll}
m_{i}^{11} & m_{i}^{12} & m_{i}^{13} & m_{i}^{14} & m_{i}^{15} \\
m_{i}^{21} & m_{i}^{22} & m_{i}^{23} & m_{i}^{24} & m_{i}^{25} \\
m_{i}^{31} & m_{i}^{32} & m_{i}^{33} & m_{i}^{34} & m_{i}^{35} \\
m_{i}^{41} & m_{i}^{42} & m_{i}^{43} & m_{i}^{44} & m_{i}^{45} \\
m_{i}^{51} & m_{i}^{52} & m_{i}^{53} & m_{i}^{54} & m_{i}^{55}
\end{array}\right)\left(\begin{array}{c}
P\left[A_{i-1}^{1}\right] \\
P\left[A_{i-1}^{2}\right] \\
P\left[A_{i-1}^{3}\right] \\
P\left[A_{i-1}^{4}\right] \\
P\left[A_{i-1}^{5}\right]
\end{array}\right)
$$

when the elements of the vectors and the matrix are shown explicitly. Because the Markov process is dealing with probabilities and the set of states is a complete one, the columns of the matrix $\mathcal{M}_{i}$ should sum up to one, i.e., $\sum_{j=1}^{5} m_{i}^{j 1}=\sum_{j=1}^{5} m_{i}^{j 2}=\cdots=\sum_{j=1}^{5} m_{i}^{j 5}=1$.

Equation (15) represents a system of equations and, for example, the fourth equation, i.e., the equation for the probability of the state $A^{4}$, reads as

$$
\begin{aligned}
P\left[A_{i}^{4}\right]= & m_{i}^{41} P\left[A_{i-1}^{1}\right]+m_{i}^{42} P\left[A_{i-1}^{2}\right]+m_{i}^{43} P\left[A_{i-1}^{2}\right]+ \\
& +m_{i}^{44} P\left[A_{i-1}^{4}\right]+m_{i}^{45} P\left[A_{i-1}^{5}\right] .
\end{aligned}
$$

By comparing this with Eq. 10 it is seen that $m_{i}^{41}=0, m_{i}^{42}=\bar{k}_{i} f_{i}, m_{i}^{43}=$ $h_{i} \bar{k}_{i} f_{i}, m_{i}^{44}=1$, and $m_{i}^{45}=0$. By inspection of Eqs. 7-11 it is straightforward to extract all the other matrix elements of the transition matrix, which gives

$$
\mathcal{M}_{i}=\left(\begin{array}{ccccc}
1 & k_{i} & h_{i} k_{i} & 0 & 0 \\
0 & \bar{k}_{i} \bar{f}_{i} & h_{i} \bar{k}_{i} \bar{f}_{i} & 0 & 0 \\
0 & 0 & \bar{h}_{i} \bar{f}_{i} & 0 & 0 \\
0 & \bar{k}_{i} f_{i} & h_{i} \bar{k}_{i} f_{i} & 1 & 0 \\
0 & 0 & \bar{h}_{i} f_{i} & 0 & 1
\end{array}\right)
$$

The transition matrix $M$ can also be form directly by examining the dynamic process shown in Fig. 3. For example, the probability of the state $A^{3}$ diminishes by an amount $(h l c+h \vec{k} \bar{f}+h \bar{k} f+\bar{h} f) P\left[A^{3}\right]$ at each time step. One is left with $1-(h k+h \bar{k} \bar{f}+h \bar{k} f+\bar{h} f)=1-(h+\bar{h} f)=\bar{h}-\bar{h} f=\bar{h}(1-f)=\bar{h} \bar{f}$, which is just the factor before $P\left[A_{i-1}^{3}\right]$ in Eq 9 .

After the transition matrices $\mathcal{M}_{i}$ are formed for each time step it is easy to calculate the probabilities of states $A^{j}$ at any time step as follows. One starts at the initial state probability vector $\mathbf{P}\left[\mathbf{A}_{0}\right]$ and multiplies this by the transition matrix $\mathcal{M}_{1}$ in order to get the probabilities $\mathrm{P}\left[\mathbf{A}_{1}\right]$ at time $t_{1}$, and 
the probabilities at all later times are obtained similarly, i.e, by multiplying previous probability vector with the corresponding transition matrix:

$$
\begin{aligned}
\mathbf{P}\left[\mathbf{A}_{1}\right] & =\mathcal{M}_{1} \mathbf{P}\left[\mathbf{A}_{0}\right] \\
\mathbf{P}\left[\mathbf{A}_{2}\right] & =\mathcal{M}_{2} \mathbf{P}\left[\mathbf{A}_{1}\right]=\mathcal{M}_{2} \mathcal{M}_{1} \mathbf{P}\left[\mathbf{A}_{0}\right] \\
& \vdots \\
\mathbf{P}\left[\mathbf{A}_{n}\right] & =\mathcal{M}_{n} \mathbf{P}\left[\mathbf{A}_{n-1}\right]=\mathcal{M}_{n} \cdots \mathcal{M}_{2} \mathcal{M}_{1} \mathbf{P}\left[\mathbf{A}_{0}\right]
\end{aligned}
$$

For example, the transition from the initial state $A_{0}$ to the state $A_{1}$ at the first time interval $t_{1}$ goes like $\left(h_{1}=0.08, k_{1}=0.95, f_{1}=1.00\right)$ :

$$
\left(\begin{array}{c}
P\left[A_{1}^{1}\right] \\
P\left[A_{1}^{2}\right] \\
P\left[A_{1}^{3}\right] \\
P\left[A_{1}^{4}\right] \\
P\left[A_{1}^{5}\right]
\end{array}\right)=\left(\begin{array}{ccccc}
1 & 0.95 & 0.076 & 0 & 0 \\
0 & 0.05 & 0.004 & 0 & 0 \\
0 & 0 & 0.920 & 0 & 0 \\
0 & 0 & 0 & 1 & 0 \\
0 & 0 & 0 & 0 & 1
\end{array}\right)\left(\begin{array}{l}
0 \\
0 \\
1 \\
0 \\
0
\end{array}\right)=\left(\begin{array}{c}
0.076 \\
0.004 \\
0.920 \\
0 \\
0
\end{array}\right) \text {. }
$$

\section{CONCLUSIONS}

In this paper, a fire risk analysis method based on the use of time-dependent event trees is presented with tentative results concerning risks to property quantified simply as the probability of structural failure. The branching probabilities describing the momentary state of the fire are obtained through Monte Carlo simulations, where statistics, computational models, and heuristic reasoning are used to determine the course of events at each simulated design fires. It is shown how to formulate the problem by using a stochastic process, which forms a Markov chain, and how to relate the transition probabilities of the stochastic process with the branching probabilities of the event trees. The method is applied to a simple example case in order to show how the method works and to clarify the connection between both approaches used to formulate the problem.

Sprinklers, smoke exhaust systems and other fire safety systems are easy to implement to the present version of the method. In future, a more realistic manual extinguishing model will be included, which also models the action of fire brigade better. In the present method the success of the fire brigade is calculated using the time passed from the ignition, whereas the arrival time of the fire brigade depends on the time of detection of the fire. The occurrence of flashover will be implemented as a separate fire scenario and the safety of life through implementation of models for life hazard and evacuation calculations.

\section{ACKNOWLEDGMENTS}

This work was carried out with a financial grant from the European Coal and Steel Community (ECSC Steel RTD Programme, Contract No.: 7210PR-251). The funding of Rautaruukki Group and Wood Focus Finland is gratefully acknowledged. 


\section{REFERENCES}

[1] Fraser-Mitchell, J.N., 'Object-Oriented Simulation (CRISP II) for Fire Risk Assessment,"Fire Safety Science - Proceedings of the Fourth International Symposium, International Association for Fire Safety Science, Boston, MA, 1994, pp. 793-804.

[2] Yung, D., Hadjisophocleous, G.V., Yager, B., "Case Study: The Use of FiRECAM $^{\mathrm{TM}}$ to Identify Cost-Effective Fire Safety Design Options for a Large 40-Storey Office Building," Proceedings of the Pacific Rim Conference and the Second International Conference on Performance-Based Codes and Fire Safety Design Methods, International Code Council, Birmingham, AL, 1998, pp. 441-452.

[3] Beck, V., “CESARE-RISK: A Tool for Performance-Based Fire Engineering Design," Proceedings of the Pacific Rim Conference and the Second International Conference on Performance-Based Codes and Fire Safety Design Methods, International Code Council, Birmingham, AL, 1998, pp. 319-330.

[4] Hietaniemi, J., Baroudi, D., Korhonen, T., Bjorkman, J., Kokkala, M., and Lappi, E. "Influence of Fire Resistance of Structures on Fire Safety in a Single-Storey Industrial Building: Risk Analysis Using a TimeDependent Event-Tree Model (in Finnish)," VTT Tiedotteita - Research Notes 2123, VTT Building and Transport, Espoo, Finland, 2002. (http://www.inf.vtt.fi/pdf/tiedotteet/2002/T2123.pdf)

[5] Alpert, R.L., "Calculation of Response Time of Ceiling-Mounted Fire Detectors," Fire Technology 8: 181-195(1972).

[6] Hasemi, Y., Wakamatsu, T., Ptchelintsev, A., "Fire Safety of Building Components Exposed to a Localized Fire," Proceedings of the ASIAFLAM 95, Interscience Communications, 1995, pp. 351-361.

[7] Myllymaki, J. and Kokkala, M., "Thermal Exposure to a High Welded I-Beam above a Pool Fire," Proceedings of the First International Workshop on Structures in Fire, University of Liege, Liege, Belgium, 2000, pp. 211-224.

[8] Paavilainen, A., "Verification of the Performance Requirements for Fire Safety in Industrial Buildings when Using Uninsulated Steel Members as Load Bearing Structure (in Finnish)," Master's Thesis, Helsinki University of Technology, Espoo, Finland, 1996.

[9] Babrauskas, V, "Burning Rates," The SFPE Handbook of Fire Protection Engineering ( $\left.2^{\text {nd }} e d\right)$, DiNenno, P.J. (ed), National Fire Protection Association, Quincy, MA, 1995, p. 3-1.

[10] Tillander, K. and Keski-Rahkonen, O., "The Influence of Fire Department Intervention to the Safety of a Building Assessed Using Fire Risk Analysis," Proceedings of the Third International Conference on Performance-Based Codes and Fire Safety Design Methods, Society of Fire Protection Engineers, Bethesda, MD, 2000, pp. 247-256. 